\title{
Research of generative ball in melting and solidification heat transfer characteristics
}

\author{
Weiguo. Wang ${ }^{1, a^{*}}$, Shuhui. Xu, Jun.You, Hao.Lu \\ ${ }^{1}$ The research centre of comprehensive and effective energy engineering \\ awangweiguo221@163.com
}

\begin{abstract}
Keywords:Phase change heat storage, paraffin ball ,natural convection ,FLUENT
\end{abstract}
Abstract. Paraffin regenerative ball physical model is presented in the paper, and the process of regenerative ball phase change heat transfer is simulated by FLUENT software. This paper expounds the temperature and liquid composition distribution in the process of the regenerative ball heat storage and heat release, compared the influence on melting and solidification in the natural convection item. Finally, this paper proposes whether natural convection item should be neglected in numerical simulation by comparing the results.

\section{Introduction}

In the shallow north area, farmers live relatively scattered, whose living standards are relatively backward, so a low investment cost and convenient maintenance of solar heating system is necessary. There is no problem to use solar energy in heating room during the sunny day, while at night, using the set thermal heating of the heat storage can also play a role [1]. So it's necessary to research the suitable program of collector- storage solar heating technology in the shallow north area.

Regenerative way is mainly divided into three categories: sensible heat regenerative, phase change heat storage and chemical heat storage. Low temperature phase change heat storage material (melting point within $0 \sim 120{ }^{\circ} \mathrm{C}$ ) commonly used in various industrial and public facilities of waste heat recovery and storage of solar energy. Because of its density energy storage, low cost, small corrosion resistance and simple manufacture, it's suitable to apply solar energy to storage heat, while paraffin is a kind of phase change heat storage material.

In order to deeply understand the mechanism of phase change heat transfer process, the paraffin phase change heat storage ball is numerical simulated in this paper, table 1 for paraffin property parameter table.

Tab.1 Physical property parameters of paraffinic

\begin{tabular}{cccccc}
\hline material & $\begin{array}{l}\text { Phase } \\
\text { transition } \\
\text { point } T(\mathrm{~K})\end{array}$ & $\begin{array}{c}\text { Phase change } \\
\text { latent heat } \\
\Delta H(\mathrm{~kJ} / \mathrm{kg})\end{array}$ & $\begin{array}{c}\text { Density } \\
\rho\left(\mathrm{kg} / \mathrm{m}^{3}\right)\end{array}$ & $\begin{array}{c}\text { specific heat } \\
\text { capacity } \\
c(\mathrm{~kJ} /(\mathrm{kg} \cdot \mathrm{K}))\end{array}$ & $\begin{array}{c}\text { thermal } \\
\text { conductivity } \\
\lambda(\mathrm{W} /(\mathrm{m} \cdot \mathrm{K}))\end{array}$ \\
\hline $\begin{array}{c}303(\text { Melting } \\
\text { Regenerati } \\
\text { ve ball }\end{array}$ & $\begin{array}{c}\text { point } \\
\begin{array}{c}300 \text { (Freezing } \\
\text { point) }\end{array}\end{array}$ & 224 & $837.7(\mathrm{~s})$ & $3.2(\mathrm{~s})$ & $0.558(\mathrm{~s})$ \\
& & $772.2(1)$ & $2.8(1)$ & $0.335(1)$ \\
\hline
\end{tabular}

\section{The physical model}

As shown in figure 1 is heat storage device model, regenerative ball piles up in the bottom of the device, while the outside air flows into the bottom of the device, flowing through the regenerative ball surface by the space between the ball and the ball.

Due to the same structure and uniform distribution of the paraffin wax ball, one is only to research and analysis process of the melting and condensation, and the physical model is shown in figure 2 . 

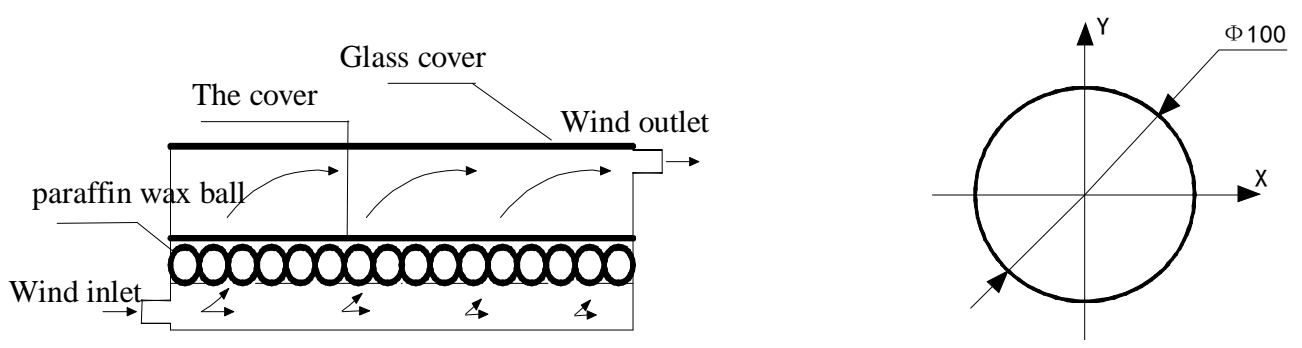

Fig. 1 Physics model of solar air collector-storage Fig .2 Physics model of phase change balls

The following assumptions for regenerative ball physical model:

A. Due to the small regenerative ball diameter, we can assume a single regenerative ball temperature uniformity in the outer wall. The outer wall is set to $310 \mathrm{k}$ when it melts, while $295 \mathrm{k}$ when it solidifies.

B. At the beginning, the ball in paraffin wax temperature is uniform and equal. The outer wall is set to $295 \mathrm{k}$ when it melts, while $310 \mathrm{k}$ when it solidifies.

C. Paraffin phase transition temperature ranges from $300 \mathrm{k}$ to $303 \mathrm{k}$, and melting heat equals solidifying heat.

D. The physical parameters of phase change material of paraffin in solid, liquid varies with temperature.

E. Due to the outer wall is a very thin layer of metal shell, large thermal conductivity and small thermal resistance, we can ignore its thermal resistance.

\section{Mathematical model}

\section{Mathematical description of considering the effects of natural convection in liquid phase:}

According to the basic theory of Solidification/Melting of FLUENT software and the simplified physical model assumptions, mathematical model can be simplified as follows [2]:

The continuity equation

$$
\frac{\partial u}{\partial x}+\frac{\partial v}{\partial y}=0
$$

Momentum conservation equation

$$
\rho\left(\frac{\partial u}{\partial \tau}+\frac{\partial u}{\partial x}+\frac{\partial v}{\partial y}\right)=-\frac{\partial p}{\partial x}+\mu\left(\frac{\partial^{2} u}{\partial x^{2}}+\frac{\partial^{2} u}{\partial y^{2}}\right)+\frac{(1-\beta)^{2}}{\left(\beta^{3}+\varepsilon\right)} A_{m u s h} \mu
$$

Energy conservation equation

$$
\rho\left(\frac{\partial v}{\partial \tau}+\frac{\partial u}{\partial x}+\frac{\partial v}{\partial y}\right)=-\frac{\partial p}{\partial y}-\rho_{l} g y+\rho_{l} g \alpha\left(T-T_{i n i}\right)+\mu\left(\frac{\partial^{2} v}{\partial x^{2}}+\frac{\partial^{2} v}{\partial y^{2}}\right)+\frac{(1-\beta)^{2}}{\left(\beta^{3}+\varepsilon\right)} A_{m u s h} v
$$

Where: $H=h+\Delta H, \quad h=h_{r e f}+\int_{T_{r e f}}^{T} c_{p} d T, \Delta H=\beta \Delta H^{\prime}$

Which $\mathrm{H}$ as the total enthalpy, $\mathrm{h}$ as part of sensible heat enthalpy, $\Delta \mathrm{H}$ as phase change latent heat. The relationship between the temperature $\mathrm{T}$ and enthalpy $\mathrm{H}$ is:

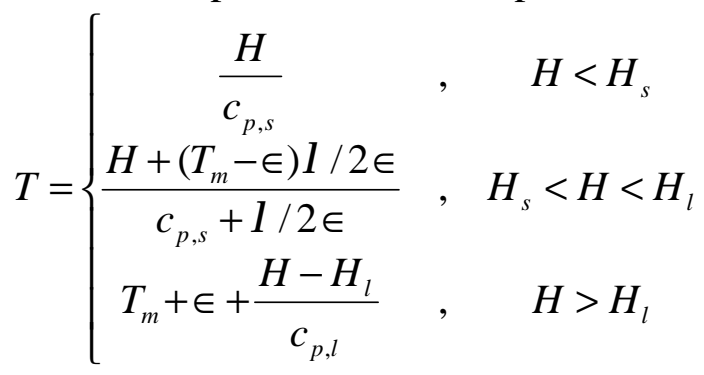




$$
\begin{aligned}
& \lambda=\left\{\begin{array}{ccc}
\lambda_{s} & , & H<H_{s} \\
\lambda_{s}+\left(T-T_{m}+\epsilon\right) \cdot \frac{\lambda_{l}-\lambda_{s}}{2 \in} & , & H_{s} \leq H \leq H_{l} \\
\lambda_{l} & , & H>H_{l}
\end{array}\right. \\
& \rho=\left\{\begin{array}{ccc}
\rho_{s} & , & H<H_{s} \\
\rho_{s}+\left(T-T_{m}+\epsilon\right) \cdot \frac{\rho_{l}-\rho_{s}}{2 \in} & , & H_{s} \leq H \leq H_{l} \\
\rho_{l} & , & H>H_{l}
\end{array}\right.
\end{aligned}
$$

Initialization conditions

$$
\left.T(x, y, \tau)\right|_{x<r, y<, \tau, 0}=T_{i n i}
$$

Boundary conditions

$$
\left.T(x, y, \tau)\right|_{x_{-} r^{r}-r, x \geq 0}=T_{w} \quad, \lambda \frac{\partial T}{\partial x}=\left.\lambda \frac{\partial T}{\partial y}\right|_{r=0}=0
$$

Mathematical description ignoring the influence of natural convection in liquid phase:

Heat storage and heat release process contains only pure heat conduction, while there is no convective heat transfer, as well no fluid velocity field, which means no momentum differential equation. Due to the space is closed, there is no quality flow into and out of, which means no mass conservation equation (or continuity equation), therefore, mathematical model including only the equation of conservation of energy. The mathematical description is shown as following.

$$
\frac{\partial H}{\partial \tau}=\lambda\left(\frac{\partial^{2} T}{\partial x}+\frac{\partial^{2} T}{\partial y}\right)
$$

In the type: $H=h+\Delta H, h=h_{r e f}+\int_{T_{r e f}}^{T} c_{p} d T, \Delta H=\beta \Delta H^{\prime}$ Which $\mathrm{H}$ as the total enthalpy, h as part of sensible heat enthalpy, $\Delta \mathrm{H}$ as phase change latent heat.

Initialization conditions

$$
\left.T(x, y, \tau)\right|_{x<r, y<r, \tau=0}=T_{i n i}
$$

Boundary conditions

$$
\left.T(x, y, \tau)\right|_{x_{-} y_{y-r}, x \geq 0}=T_{w} \quad \lambda \frac{\partial T}{\partial x}=\left.\lambda \frac{\partial T}{\partial y}\right|_{\gamma\lrcorner 0}=0
$$

\section{Results and analysis of numerical simulation calculation}

\section{The heat transfer characteristics in the process of phase transition:}

In the melting process, the initial temperature is set to $290 \mathrm{k}$, while the wall temperature is set to $310 \mathrm{k}$. The characteristics of phase change materials heat transfer in the heat storage phase change are shown in Fig.3 and Fig.4.

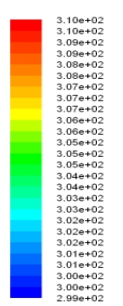

(considering natural convection)

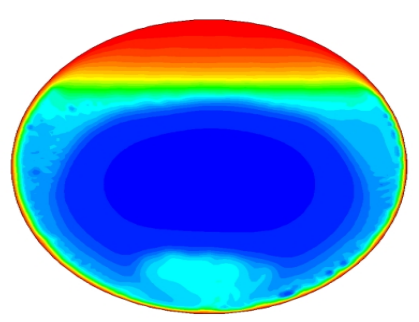

Fig.3 Temperature distribution of heat storage ball at 500s in the melting process

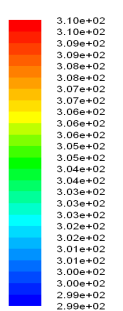

(neglecting the natural convection)

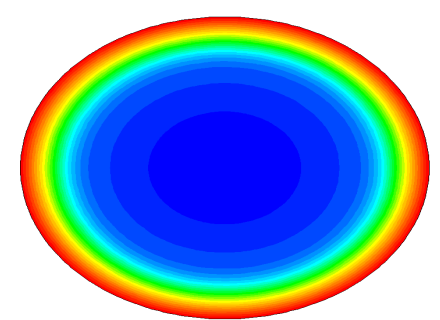




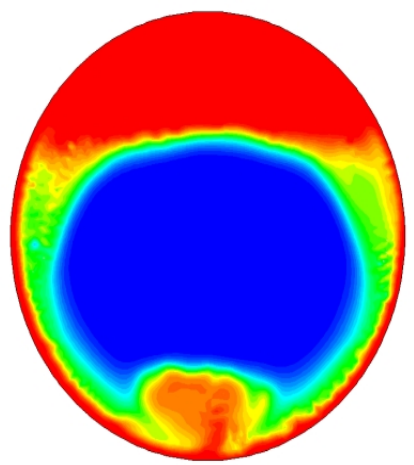

(considering natural convection)

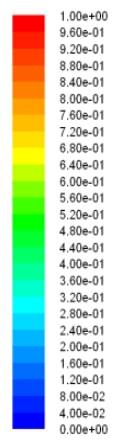

(neglecting the natural convection)

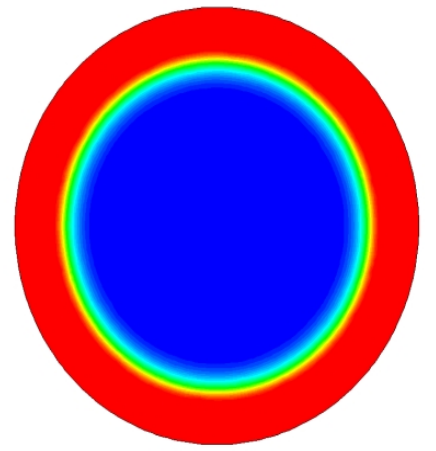

Fig.4 Proportion of solid-liquid phase of heat storage ball at 500s in the melting process

In the solidification process, the initial temperature is set to $310 \mathrm{~K}$, while the wall temperature is set to $295 \mathrm{~K}$. The characteristics of phase change materials heat transfer in the heat storage phase change are shown in Fig.5 and Fig.6.

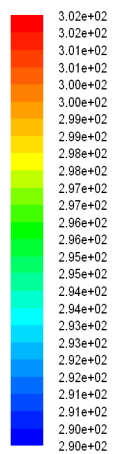

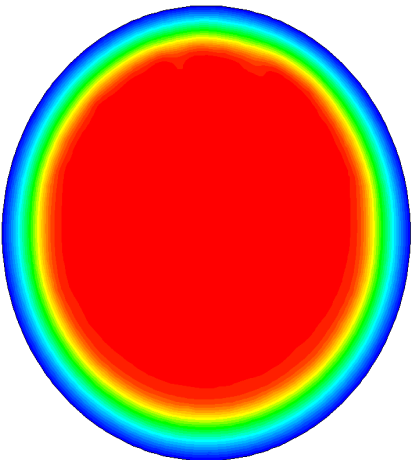

(considering natural convection)
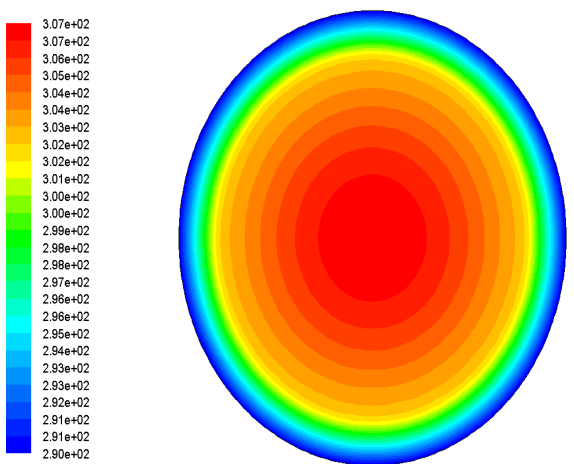

(neglecting the natural convection)

Fig.5 Temperature distribution of heat storage ball at 200s in the solidification process
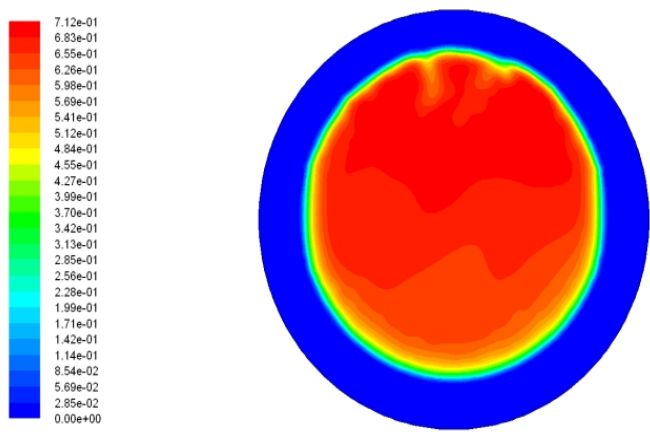

(considering natural convection)
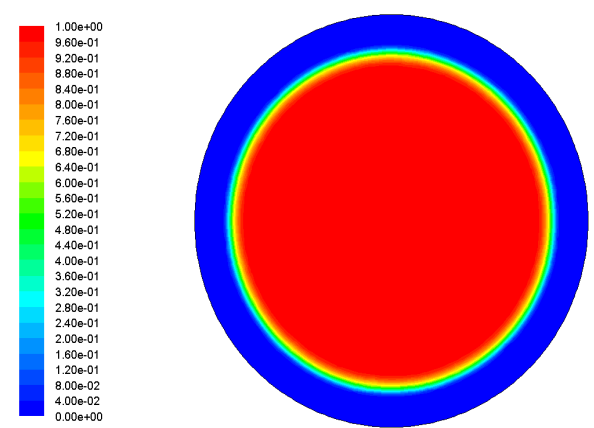

Fig.6 Proportion of solid-liquid phase of heat storage ball at 200s in the solidification process

The temperature field and the rule of the mobile phase interface in melting process

A. Change curve of average temperature of PCM in heat storage ball with time increasing

The wall temperature of regenerative ball is $310 \mathrm{~K}$, the initial temperature as $290 \mathrm{~K}$. Figure. 7 are the simulation results of temperature change with the iteration time of paraffin inside ball, respectively considered paraffin liquid natural convection and without considering paraffin liquid under the condition of natural convection. 


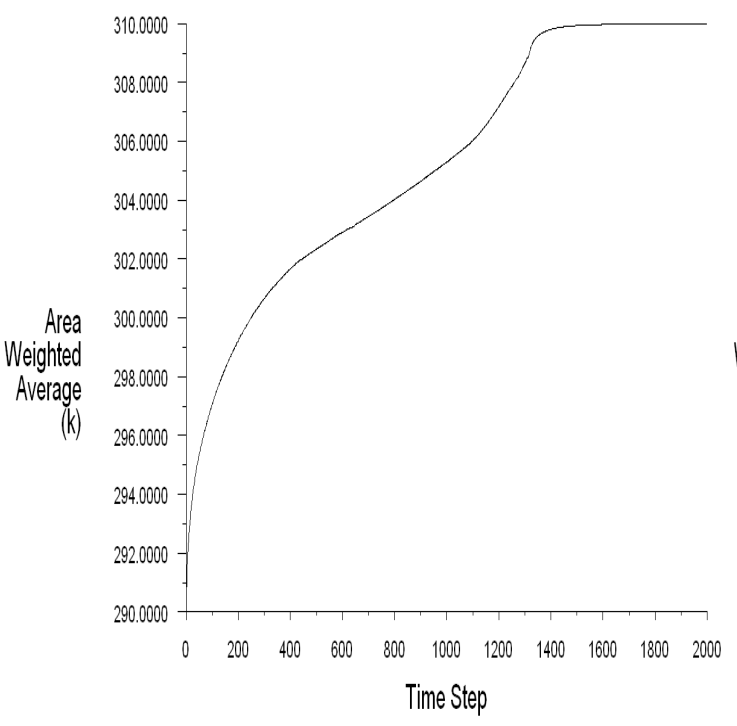

(considering natural convection)

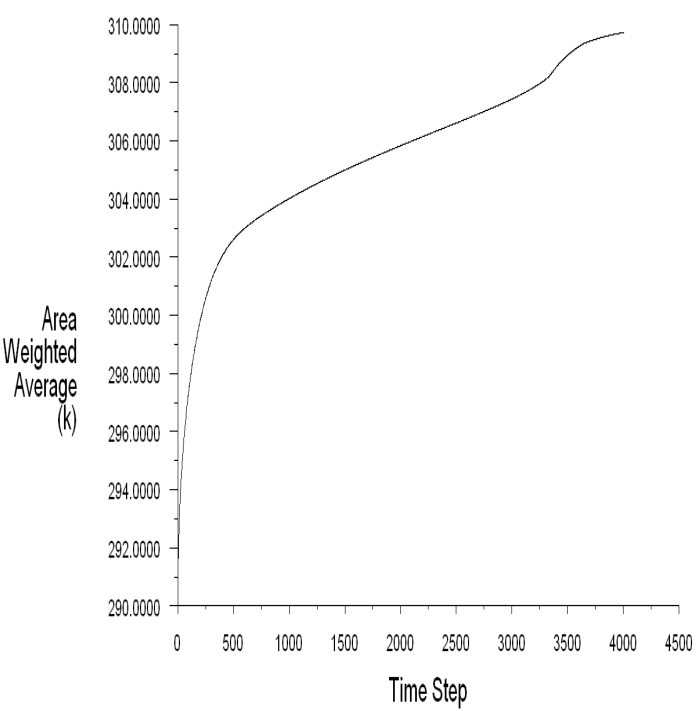

(neglecting the natural convection)

Fig.7 Change curve of average temperature of PCM in melting process

Considering natural convection, in the initial stage, because thermal conductivity is dominant in heat transfer inside the ball, the heat transfer area is larger, the hot resistance is small and heat transfer capacity is large, the ball paraffin wax will heat up fast in a short time; The increasing of the liquid working medium, transferring heat through the liquid, thermal resistance increases gradually, melting rate dropping, but this time there are both heat conduction and convection, so the heat transfer capacity is still increasing, and temperature continues to rise; Completely melt with the final temperature $310 \mathrm{k}$.

Ignoring the natural convection, temperature increases rapidly at first as well as figure3-19. The process is shorter, about $500 \mathrm{~s}$, generally the same time considering natural convection in liquid phase, which illustrates the natural convection of liquid phase have less influence on the heat transfer at the beginning of the melting; In middle stage of melting process, the average temperature of the paraffin wax inside the ball changes into the linear incremental, while the process costs a long time; In the final stages, it gradually increases until $310 \mathrm{k}$ in a short time.

\section{B. The proportion change with iteration time step of paraffin liquid in ball:}

Figure. 5 is the simulation results of paraffin liquid ratio change with iteration time step, respectively considering paraffin liquid natural convection and without considering the natural convection.

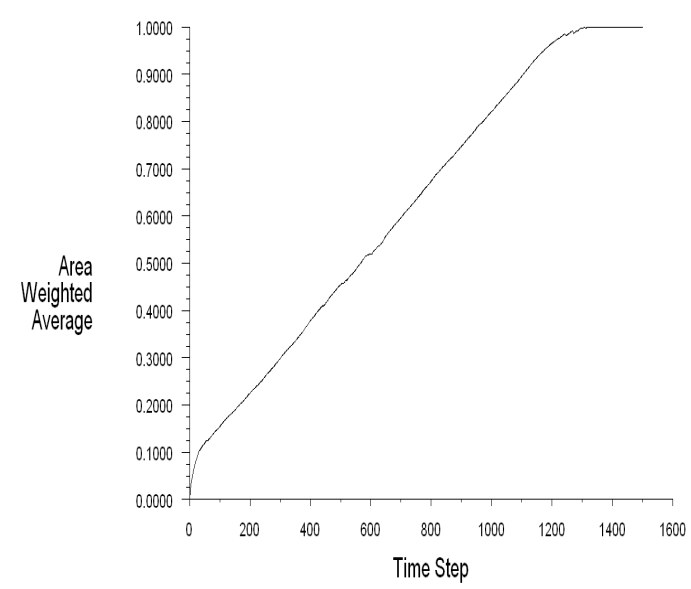

(considering natural convection)

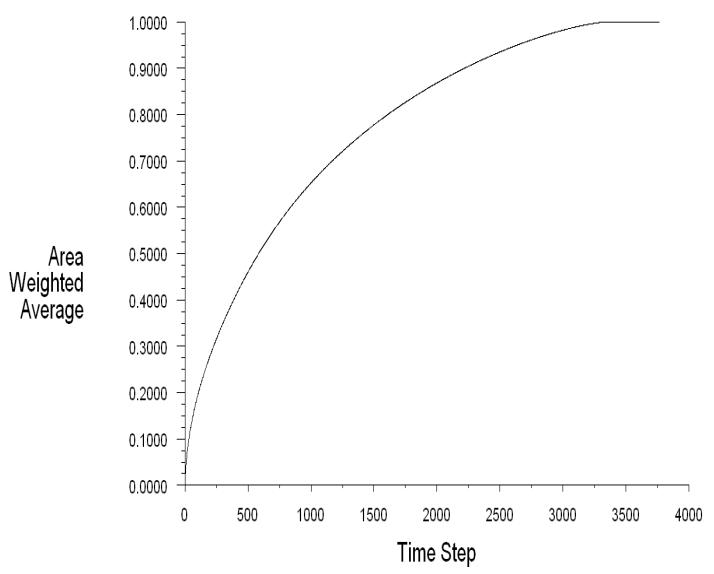

(neglecting the natural convection)

Fig. 8 Change curve of liquid phase proportion in melting process 
Considering natural convection in liquid phase, at the initial stage, due to the heat transfer area is larger, the hot resistance is small and the heat is larger, the paraffin wax melts rapidly in a short time; Liquid proportion is larger and larger, so the liquid paraffin wax produced significantly by natural convection, and this phase of liquid ratio curve changes linearly; The last liquid ratio increased to 1.0 , melted completely.

Ignoring the natural convection in liquid phase, the whole melting process stage has no continuous linear change, due to ignoring the natural convection in liquid phase and the whole melting process is considered to be pure heat conduction process.

\section{The temperature variation and boundary move rule of solidification process.}

\section{A. The average temperature variation of paraffin in the ball with iterative time step:}

The wall temperature of thermal storage ball is $295 \mathrm{~K}$, and the initial temperature is $310 \mathrm{~K}$. Fig.9 considers the simulation result, that the change of average temperature of the paraffin wax is along with time when paraffin liquid is in natural convection and another is not.

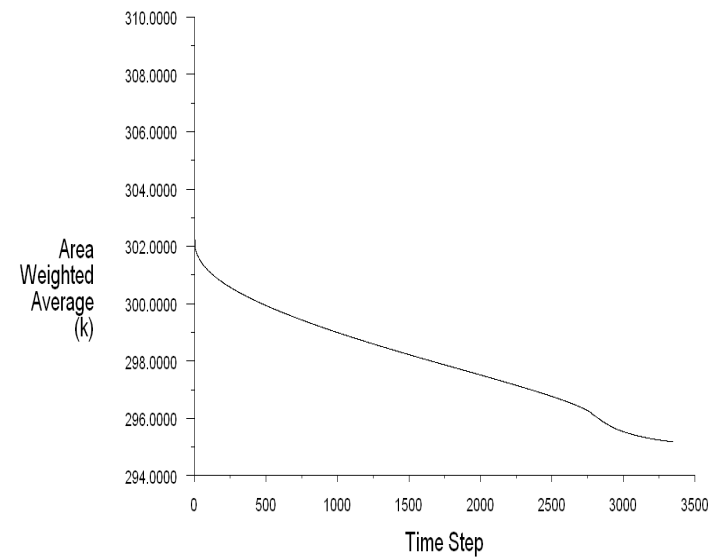

(considering natural convection)

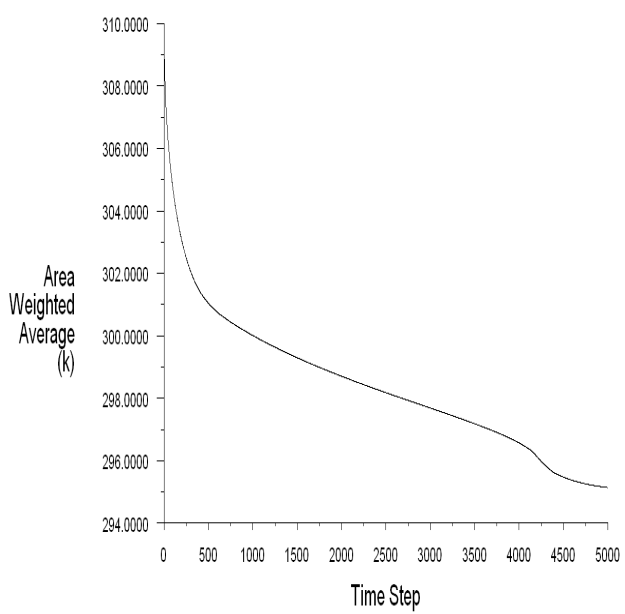

(neglecting the natural convection)

Fig.9 Change curve of average temperature of PCM in solidification process

In the Fig.9, natural convection influences the solidification slightly, and the changing curve of temperature in the process is basically the same. The considering natural convection one of Fig.9 showed in Fig.10 as follows that shows the enlargement picture of initial phase of the solidification heat release.

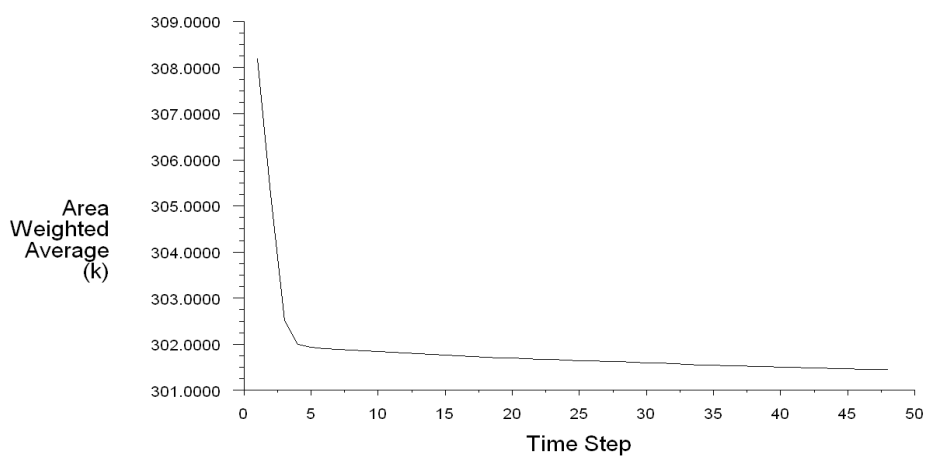

Fig.10 Enlargement picture in part of the Figure 9

When the solidification process starts, the liquid phase is less meantime the heat transfer is the main heat conduction and the average temperature of the heat storage ball drops quickly. Then it's time to enter the phase change stage, the temperature decreased slowly in the phase change and this stage is almost flat straight down, continuing for a long time because of the latent heat and heat release process requiring a longer. In the final stage, the average temperature of paraffin wax decreases rapidly, which is due to the lower limit of the temperature range of the ball, which can quickly release the remaining sensible heat, but because of the solidified medium thickness and the 
heat release rate of import blocked, the rate of sensible heat decreased gradually until the ball paraffin temperature has reached $295 \mathrm{~K}$, namely the end of solidification heat.

\section{B. The liquid phase variation of paraffin in the ball with iterative time step}

Fig.11 shows the changing curve of liquid phase proportion when considering and neglecting the convection in the heat storage ball with time increasing respectively.

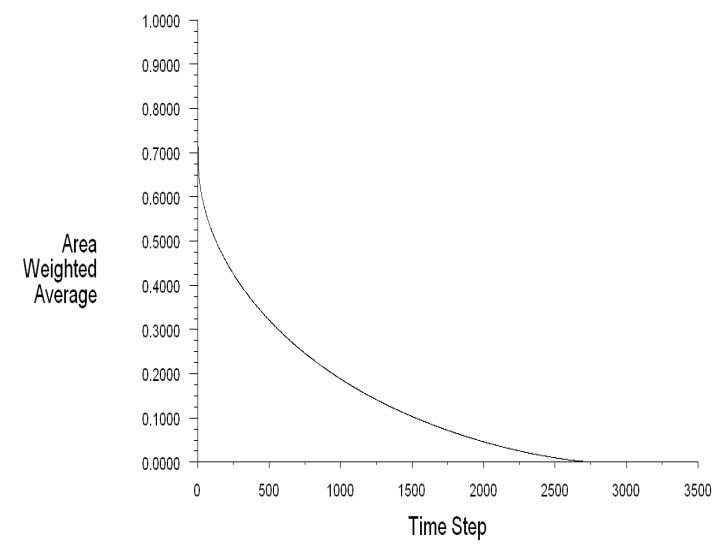

(considering natural convection)

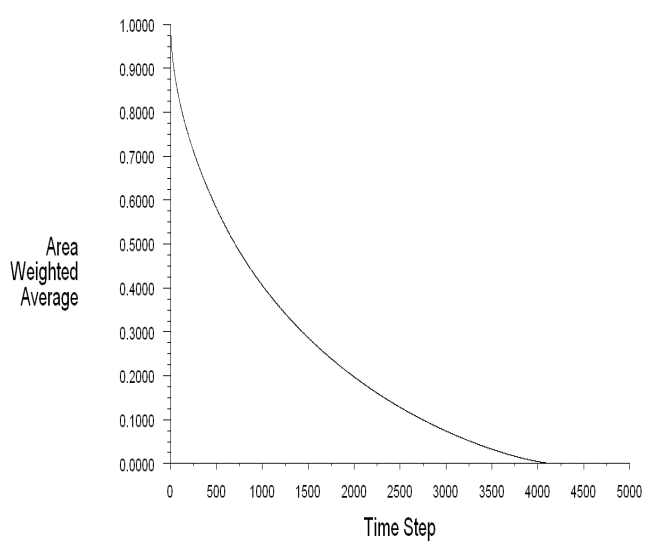

(neglecting the natural convection)

Fig.11 Change curve of liquid phase proportion in solidification process

It can be seen that the natural convection of liquid phase in the left one of Figure.11, the ratio of liquid phase decreases rapidly in the initial stage of the melting process meantime the phase boundary is moving rapidly from the outside to the inner surface; The thickness of solid material is increasing, while the thermal conductivity of solid paraffin is higher than that of liquid phase, but the thickness and the thermal resistance is increasing, which leads to less heat transfer, and the solidification rate is decreasing. At last the paraffin wax solidified totally.

When the natural convection of liquid phase is neglected, the variation of the liquid phase ratio of paraffin in the ball with the iteration time step is shown in the right of Figure.11. Comparing the left one, the shape of the liquid phase change curve is basically the same, but the time of solidification is different.

\section{Conclusion}

The effect of natural convection on the melting process is slightly larger than that of the solidification process.

When melting heat storage, the natural convection in the liquid phase is considered or not and the proportion of the liquid phase and the weighted average temperature vary greatly with the iterative time step.

The natural convection in the liquid phase has little effect on the solidification without considering the time of solidification in the natural convection when the solidification is releasing heat.

\section{Acknowledgement}

Project supported by Energy and Power Engineering Specialty Construction and Beijing Municip ality Key Lab of Heating, Gas Supply, Ventilating and Air Conditioning Engineering. 


\section{References}

[1] Donghua Peng,Zhenqian Chen,Mingheng Shi. Solar panel collector/energy storage phase change heat transfer numerical simulation. Journal of solar energy, 2009, 30(7): 911-915.

[2] B.Pause.Textile Testing \& Innovation Longmont CO/ USA Possibilities for Air-Conditioning Buildings With Phase Change Material. International textile guides, 2001,15(2): 55-58. 